Preprint typeset using $\mathrm{LAT}_{\mathrm{E}} \mathrm{X}$ style emulateapj v. 5/2/11

\title{
ON GALACTIC DENSITY MODELING IN THE PRESENCE OF DUST EXTINCTION
}

\author{
Jo Bovy ${ }^{1,2,3}$, Hans-Walter Rix ${ }^{4}$, Gregory M. Green ${ }^{5}$, Edward F. Schlafly ${ }^{4}$, And Douglas P. Finkbeiner ${ }^{5}$
}

\begin{abstract}
Inferences about the spatial density or phase-space structure of stellar populations in the Milky Way require a precise determination of the effective survey volume. The volume observed by surveys such as Gaia or near-infrared spectroscopic surveys, which have good coverage of the Galactic midplane region, is highly complex because of the abundant small-scale structure in the three-dimensional interstellar dust extinction. We introduce a novel framework for analyzing the importance of smallscale structure in the extinction. This formalism demonstrates that the spatially-complex effect of extinction on the selection function of a pencil-beam or contiguous sky survey is equivalent to a lowpass filtering of the extinction-affected selection function with the smooth density field. We find that the angular resolution of current 3D extinction maps is sufficient for analyzing Gaia sub-samples of millions of stars. However, the current distance resolution is inadequate and needs to be improved by an order of magnitude, especially in the inner Galaxy. We also present a practical and efficient method for properly taking the effect of extinction into account in analyses of Galactic structure through an effective selection function. We illustrate its use with the selection function of red-clump stars in APOGEE using and comparing a variety of current 3D extinction maps.

Subject headings: dust, extinction — Galaxy: kinematics and dynamics — Galaxy: structure — methods: data analysis — stars: statistics — surveys
\end{abstract}

\section{INTRODUCTION}

A common problem in the analysis of stellar surveys in the Milky Way is to account for the effects of astrophysical and procedural selection effects on the manner in which the Galactic volume is surveyed (e.g., Rix \& Bovy 2013). The latter effects primarily consist of the fraction of stars observed as a function of their position on the celestial sphere, stellar type, color, or magnitude. Examples of the astrophysical effects include how interstellar extinction impacts the observed magnitudes of stars or the manner in which different stellar types trace the underlying stellar populations. Accounting for these selection biases is essential for using stellar surveys to determine the spatial or phase--space distribution of stars in our Galaxy.

The basic formalism for fitting models of the density or phase-space distribution has been reviewed by Rix \& Bovy (2013). The formalism presented there is general, but in this paper we focus on understanding the formalism and its implementation in the case that the effect of interstellar extinction on the selection function of a stellar survey is significant. To motivate this, Figure 1 displays the amount of interstellar extinction to $5 \mathrm{kpc}$ over the whole sky from a combination of the Marshall et al. (2006), Green et al. (2015), and Drimmel et al. (2003) extinction maps (see the Appendix). It is clear from this map that any investigation of the phase--space distribution of the stellar disk near

1 Department of Astronomy and Astrophysics, University of Toronto, 50 St. George Street, Toronto, ON, M5S 3H4, Canada; bovy@astro.utoronto.ca

2 Institute for Advanced Study, Einstein Drive, Princeton, NJ 08540, USA

3 John Bahcall Fellow

4 Max-Planck-Institut für Astronomie, Königstuhl 17, D69117 Heidelberg, Germany

5 Harvard-Smithsonian Center for Astrophysics, 60 Garden Street, Cambridge, MA 02138, USA the midplane is strongly affected by the highly filamentary structure of the extinction. Determining simple cuts to define a volume-complete sample of stars in this region of the Galaxy is difficult and sub-optimal.

Below, we present a simple and efficient formalism for determining and understanding the effect of extinction on the effective volume of a survey. We do this for two cases: (a) a large-area survey such as Gaia that covers a significant fraction of the sky and (b) a pencil-beam survey, such as the spectroscopic APOGEE survey (S. R. M. Majewski, et al., 2015, in preparation), consisting of a number of small-area field pointings. While the formalism is different in practice, in both cases an important conclusion is that the effect of extinction on small spatial scales is unimportant when considering smooth models for the density or phase-space distribution of stars.

The outline of this paper is as follows. $\S 2$ reviews the basic likelihood-based formalism for inferring stellar phase-space distributions. We present the formalism for determining the effective survey volume necessary in the likelihood for large-area surveys and pencil-beam surveys in $\S \S 3.1$ and 4.1, respectively. We give a detailed example of a Gaia-like large-area survey of red-clump (RC) stars in $\S 3.2$. $\S 4.2$ discusses the effect of extinction on the pencil-beam APOGEE volume selection of RC stars as an example of the formalism in $\S 4.1$. We discuss and conclude in $\S 5$.

\section{LIKELIHOOD-BASED DENSITY MODELING AND THE EFFECTIVE SURVEY VOLUME}

As discussed in detail by Bovy et al. (2012) and Rix \& Bovy (2013), the correct model of star counts is an inhomogeneous Poisson process. This process is characterized by a rate function $\lambda(O \mid \theta)$ that specifies the model prediction for the number of stars as a function of $O=(l, b, D, m, c,[\mathrm{Fe} / \mathrm{H}])$; the model is parameterized by parameters $\theta$. Here we have written the observables in a generic form consisting of: (a) the position 


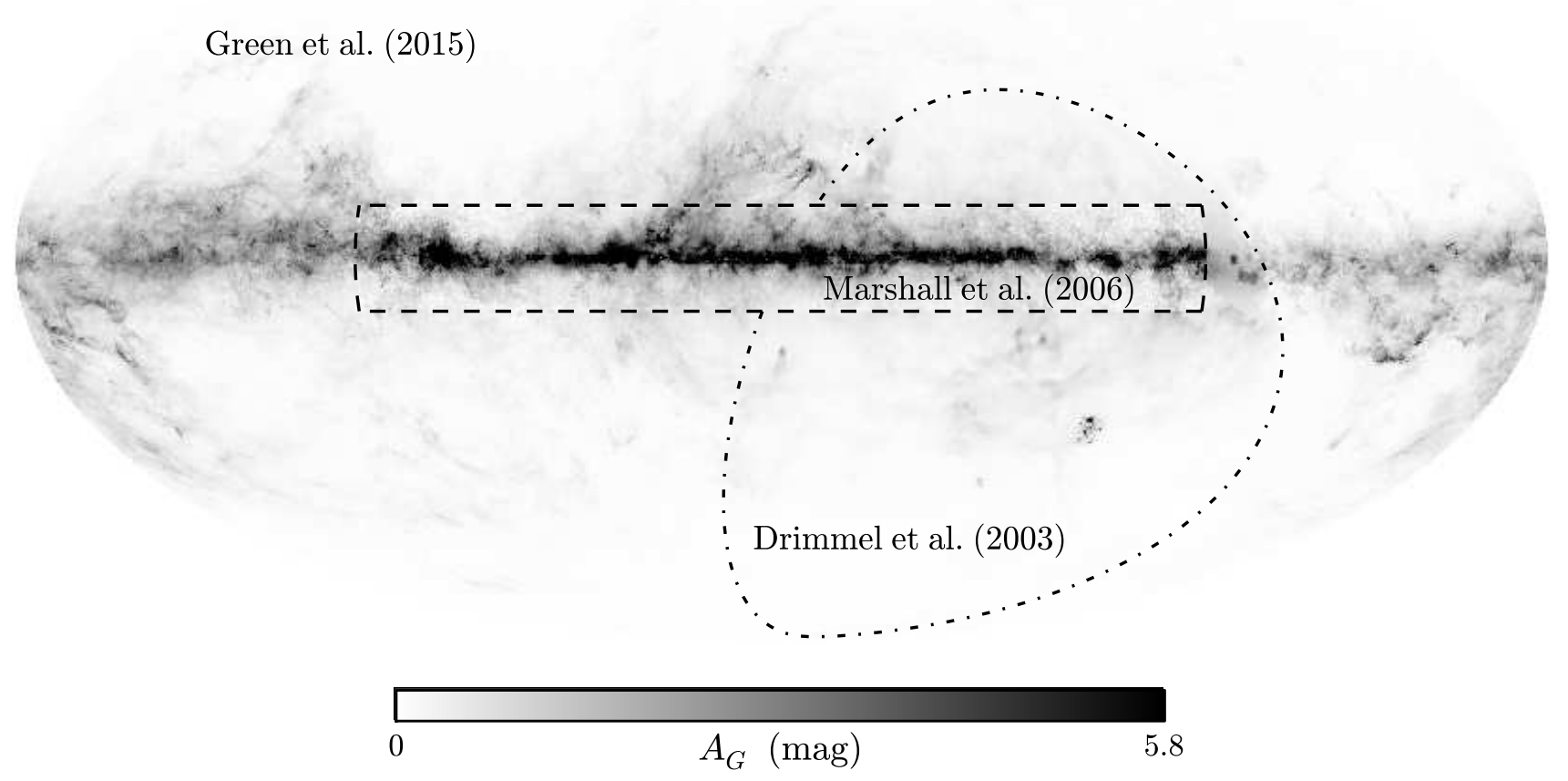

FIG. 1. - $G$-band extinction to $5 \mathrm{kpc}$ from the Sun, saturated at the approximate maximum $A_{G}$ for which we can see the RC at $G<20$ (that is, in Gaia). For the APOGEE $H$-band observations, this extinction map saturates at $A_{H} \approx 1.1$, which is the maximum extinction to which the $\mathrm{RC}$ can be seen to $\approx 4.5 \mathrm{kpc}$ in medium-deep APOGEE observations. The boundaries of the three extinction maps that are combined to obtain full-sky coverage (see the Appendix) are indicated. Much of the Galactic plane region is too extinguished for Gaia or APOGEE to be able to see the $\mathrm{RC}$ at $\approx 5 \mathrm{kpc}$ and larger distances.

in Galactic longitude, latitude, and heliocentric distance $(l, b, D),(\mathrm{b})$ an apparent magnitude $m$, and (c) a color $c$ and metallicity $[\mathrm{Fe} / \mathrm{H}]$. It is necessary to include the distribution of color (or multiple colors) and potentially metallicity when the absolute magnitude $M$ of the stellar tracer being considered depends on these. We will see below that the formalism is especially simple in the case of a standard candle, that is, a stellar tracer for which $M$ is constant; however it is straightforward to include the full dependence of $M$ on color(s) and metallicity, or any other observable that affects the absolute magnitude.

The rate function $\lambda(O \mid \theta)$ is given by

$$
\begin{aligned}
\lambda(O \mid \theta) & =\nu_{*}(X, Y, Z \mid \theta) \times|J(X, Y, Z ; l, b, D)| \\
& \times \rho(M, c,[\mathrm{Fe} / \mathrm{H}] \mid X, Y, Z) \times S(l, b, m),
\end{aligned}
$$

where $\nu_{*}(\cdot \mid \theta)$ is the spatial density in Galactocentric rectangular coordinates $(X, Y, Z)$ that we are ultimately most interested in and that depends on parameters $\theta,|J(X, Y, Z ; l, b, D)|=D^{2} \cos b$ is the Jacobian of the transformation between $(X, Y, Z)$ and $(l, b, D)$, $\rho(M, c,[\mathrm{Fe} / \mathrm{H}] \mid X, Y, Z)$ is the density of stars in absolutemagnitude-color-metallicity space (normalized to integrate to one), and $S(l, b, m)$ is the survey selection function (the fraction of stars from the underlying population of potential targets observed by the survey). For simplicity we have assumed that the survey selection function does not depend on color, metallicity, or any other ob- servable, but including these is straightforward.

As in Bovy et al. (2012), the rate has an additional amplitude parameter that gives the number density of stars at a reference location. The full log likelihood for the Poisson process is

$$
\ln \mathcal{L}(\theta)=\sum_{i} \ln \lambda\left(O_{i} \mid \theta\right)-\int \mathrm{d} O \lambda(O \mid \theta) .
$$

The integral in this equation is the expected number of stars given the model parameters that provides the normalization of the rate likelihood. It does not depend on the individual data point, but is instead a property of the whole survey for a given model specified by $\theta$. In what follows we refer to this integral as the effective volume $V_{\text {eff }}$ of the survey, because it is proportional (up to a constant, the inverse local density) to the traditional definition of the effective volume.

In many cases, the overall amplitude parameter is uninteresting. To remove the amplitude parameter from further consideration, we can marginalize the probability of the parameters of the rate function over the amplitude of the rate. Using a prior on the amplitude that is a power-law with exponent $\alpha$, the marginalized likelihood is given by

$$
\ln \mathcal{L}(\theta)=\sum_{i} \ln \left[\frac{\lambda\left(O_{i} \mid \theta\right)}{V_{\text {eff }}}\right]-(1+\alpha) \ln V_{\text {eff }} .
$$


For a prior that is inversely proportional to the amplitude $(\alpha=-1)$, the second term in this equation is zero. The likelihood can be simplified to

$$
\ln \mathcal{L}(\theta)=\sum_{i} \ln \left[\frac{\nu_{*}\left(X_{i}, Y_{i}, Z_{i} \mid \theta\right)}{V_{\mathrm{eff}}}\right]-(1+\alpha) \ln V_{\mathrm{eff}}
$$

when the rate $\lambda\left(O_{i} \mid \theta\right)$ only depends on $\theta$ through $\nu_{*}(\cdot \mid \theta)$, which is often a good assumption. Then $\nu_{*}\left(X_{i}, Y_{i}, Z_{i} \mid \theta\right)$ is the only factor in $\lambda\left(O_{i} \mid \theta\right)$ that depends on $\theta$; the other factors can be dropped.

It is the effective volume that is difficult to compute in the presence of filamentary extinction and it is the focus of the remainder of this paper.

\section{THE EFFECTIVE SURVEY VOLUME FOR LARGE-AREA SURVEYS}

\subsection{Formalism}

In this section, we work out the formalism for calculating the effective survey volume in the presence of dust for a contiguous survey of a large part of the sky. This is useful for analyzing the spatial structure of the Milky Way from photometric surveys or for analyzing the full phase-space structure from Gaia data. We assume that the conversion between distance and absolute magnitude can be written as a function of an unreddened color and metallicity, although for a photometric survey the latter might be replaced by a second color or dropped entirely. We also assume that the selection function is only a function of magnitude - we denote this magnitude in this section by $G$ with Gaia in mind-although the generaliza- tion to include color dependence (whether corrected for extinction or not) is straightforward. For contiguous surveys the selection function typically does not explicitly depend on sky position and we write it as $S(G[\cdot])$, although it could be easily incorporated in what follows ${ }^{6}$; we use the argument $G[\cdot]$ to indicate the dependence of $G$ on $(l, b, D, c,[\mathrm{Fe} / \mathrm{H}])$, but we do not always write these variables explicitly to keep the expressions cleaner. We first write out the integration over $O$ in the effective volume in equation (4) explicitly

$$
\begin{aligned}
\int \mathrm{d} O \lambda(O \mid \theta)= & \iiint \mathrm{d} l \mathrm{~d} b \mathrm{~d} D D^{2} \cos b\left[\nu_{*}(X, Y, Z \mid \theta)\right. \\
& \left.\times \iint \mathrm{d} c \mathrm{~d}[\mathrm{Fe} / \mathrm{H}] \rho(c,[\mathrm{Fe} / \mathrm{H}]) S(G[\cdot])\right],
\end{aligned}
$$

where we have used that $|J(X, Y, Z ; l, b, D)|=D^{2} \cos b$. The integral over $(c,[\mathrm{Fe} / \mathrm{H}])$ is the integration over the absolute-magnitude distribution and it can be efficiently computed using Monte Carlo integration (see below). We can then write the density $\nu_{*}(X, Y, Z \mid \theta)^{7}$ as $\nu_{*}(l, b, D \mid \theta)$ and write its dependence on $(l, b)$ using an orthonormal basis expansion, say in terms of spherical harmonics $Y_{\ell}^{m}(\pi / 2-b, l)$

$$
\nu_{*}(l, b, D \mid \theta)=\sum_{\ell=0}^{\infty} \sum_{m=-\ell}^{\ell} \nu_{*, \ell m}(D \mid \theta) Y_{\ell}^{m}(\pi / 2-b, l) .
$$

Then the effective volume becomes

$$
\int \mathrm{d} O \lambda(O \mid \theta)=\sum_{\ell=0}^{\infty} \sum_{m=-\ell}^{\ell} \int \mathrm{d} D D^{2} \nu_{*, \ell m}(D \mid \theta) \times \iint \mathrm{d} c \mathrm{~d}[\mathrm{Fe} / \mathrm{H}] \rho(c,[\mathrm{Fe} / \mathrm{H}]) \times \iint \mathrm{d} l \mathrm{~d} b \cos b Y_{\ell}^{m}(\pi / 2-b, l) S(G[\cdot]),
$$

(where all of the integrations are nested, not independent) and we can introduce the effective selection function $\boldsymbol{S}_{\ell m}$ of order $(\ell, m)$

$$
\begin{aligned}
\boldsymbol{S}_{\ell m}(D)= & \iint \mathrm{d} c \mathrm{~d}[\mathrm{Fe} / \mathrm{H}] \rho(c,[\mathrm{Fe} / \mathrm{H}]) \\
& \times \iint \mathrm{d} l \mathrm{~d} b \cos b Y_{\ell}^{m}(\pi / 2-b, l) S(G[\cdot]) .
\end{aligned}
$$

(general case; 8)

If $\rho(c,[\mathrm{Fe} / \mathrm{H}])$ depends on $(l, b, D)$, then that dependence can be incorporated by moving the integration over $\rho(c,[\mathrm{Fe} / \mathrm{H}] \mid l, b, D)$ into the integral over $(l, b)$. For a stan-

\footnotetext{
6 The boundaries of a partial-sky, contiguous survey can be incorporated by restricting the integration area in $(l, b)$ below to that of the survey, although sharp survey boundaries should be apodized to avoid ringing in the basis-function expansion below

7 When analyzing the full six-dimensional phase-space distribution this density is replaced by the integration of the $6 \mathrm{D}$ DF over velocity, but the formalism given here still applies when the selection function is independent of kinematics (which it typically is).
}

dard candle, equation (8) simplifies to

$$
\boldsymbol{S}_{\ell m}(D)=\iint \mathrm{d} l \mathrm{~d} b \cos b Y_{\ell}^{m}(\pi / 2-b, l) S(G[l, b, D]) .
$$

(standard candle; 9)

The effective volume in terms of $\boldsymbol{S}_{\ell m}$ becomes

$$
\int \mathrm{d} O \lambda(O \mid \theta)=\int \mathrm{d} D D^{2} \sum_{\ell=0}^{\infty} \sum_{m=-\ell}^{\ell} \nu_{*, \ell m}(D \mid \theta) \boldsymbol{S}_{\ell m}(D) .
$$

Equation (10) makes it clear that the effective survey volume is essentially the cross-correlation of the density with the effective selection function. Because the Galactic stellar phase-space density is much smoother than the extinction map, this cross correlation is essentially a lowpass filtering of the extinction-affected selection function with the density that limits the influence of the smallscale power in the interstellar dust distribution.

We discuss the precision to which the effective survey volume can be computed as a function of the angular and distance resolution in the $3 \mathrm{D}$ extinction map below. The 


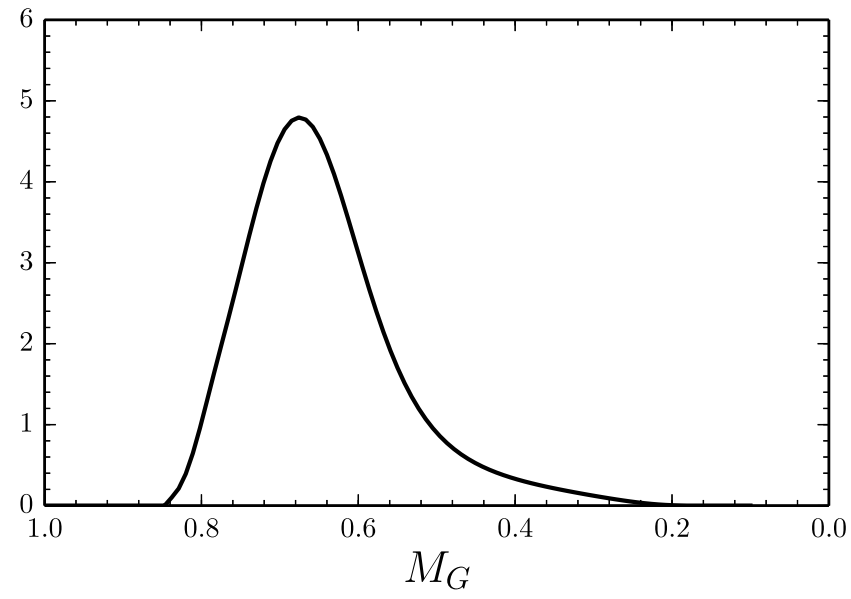

FIG. 2.- Distribution of the absolute $G$-band magnitude derived from PARSEC isochrones for an RC sample selected using the cuts from Bovy et al. (2014) and assuming a solar-neighborhood metallicity distribution. The distribution of $M_{G}$ peaks at $M_{G}=0.68$ with an approximate width of 0.08 mag.

required angular resolution depends on the sample size, the complexity of the model, the sky coverage, etc. and it can be determined from an analysis of the cross correlation in equation (10) as in the example below. Once a good angular resolution is determined one will in practice prefer to work with the effective selection function in position space. For this we define the effective selection function $\mathbf{s}(l, b, D)$

$$
\boldsymbol{s}(l, b, D)=\iint \mathrm{d} c \mathrm{~d}[\mathrm{Fe} / \mathrm{H}] \rho(c,[\mathrm{Fe} / \mathrm{H}]) S(G[\cdot]),
$$

(general case; 11 )

which can be efficiently computed using Monte-Carlo integration. For a standard candle we simply have that $\boldsymbol{s}(l, b, D)=S(G[\cdot])$. In both cases the $(l, b)$ dependence of $\mathbf{S}(l, b, D)$ is through the $(l, b)$ dependence of the extinction that gives $G=M_{G}+\mu+A_{G}(l, b, D)$, where $M_{G}$ is the absolute magnitude and $\mu$ is the distance modulus. The effective volume is then simply

$$
\begin{aligned}
& \int \mathrm{d} O \lambda(O \mid \theta)= \\
& \qquad \iint \mathrm{d} l \mathrm{~d} b \mathrm{~d} D D^{2} \cos b \nu_{*}(X, Y, Z \mid \theta) \boldsymbol{\sigma}(l, b, D) .
\end{aligned}
$$

Thus, in practice we do not need to work in Fourier space, but the Fourier space analysis is helpful to elucidate the importance of small-scale power in the extinction map and to determine an appropriate resolution to discretize the integration in equation (12).

\subsection{Gaia example}

In this section we work through an example of the formalism described above to determine the impact of interstellar extinction on phase-space inferences from Gaia. We assume that the stellar tracers for which we are fitting the density distribution are RC stars, which are close to the standard candle case. For the purpose of this example, we estimate the absolute magnitude distribution of RC stars in the Gaia $G$ band as follows. We use PARSEC isochrones (Bressan et al. 2012) and the transformation between $(g, g-z)$ and $G$ from Jordi et al. (2010) to determine the mean absolute magnitude of stars of a given age and overall metallicity $[\mathrm{Fe} / \mathrm{H}]$ that satisfy the RC selection criteria of Bovy et al. (2014). We find that the spread at a given age and metallicity is small and furthermore that the age dependence is small. Therefore, we determine $M_{G}([\mathrm{Fe} / \mathrm{H}])$ as the mean $M_{G}$ at an age of 5 Gyr (thus effectively ignoring the color dependence in the integrals above). We use the local metallicity distribution (Hayden et al. 2015) to determine the distribution of $M_{G}$ from this. The resulting distribution of $M_{G}$ is displayed in Figure 2. The distribution peaks at $M_{G}=0.68$ and is narrow with an approximate width of 0.08 mag. We stress that this is a simplified treatment of the absolute magnitude distribution, which will in detail depend on the position in the Galaxy through its dependence on age and metallicity. This dependence can be taken into account using the formalism above. We use 10,000 Monte Carlo samples from the distribution in Figure 2 to approximate the integration over the intrinsic absolute magnitude distribution above.

We consider a Gaia-like survey of RC stars, with a selection function that is constant over the full sky between $G=3$ and $G=20$ and compute the effective volume for a density model that consists of a radial and vertical exponential disk with a scale length of $3 \mathrm{kpc}$ and a scale height of $300 \mathrm{pc}$. The ingredients of the effective areathe angular part of the integration in equation (10) at two different distances are shown in Figure 3. The top panels display the angular power spectra of all of the ingredients: the density distribution, the extinction distribution, and the effective selection function of equation $(8)^{8}$. While most of the power in the extinction map is on large scales, the power on smaller scales only slowly diminishes. The red line that shows the angular power spectrum of the effective selection function demonstrates that most of the power in the extinction map is transferred to $\mathbf{S}$. However, the density of a typical exponential disk has a much stronger decline in power toward small scales. The middle panels display the cross power spectrum between the extinction map and the density as well as that between $\mathbf{S}$ and the density. As expected, on large scales where the density power spectrum is close to constant, the cross power spectrum follows that of the extinction or $\mathbf{S}$, but on small scales the power is significantly reduced due to the lack of small-scale power in the density. The bottom panels of Figure 3 show the impact of the resolution of the extinction map when computing the effective survey volume. These panels display the er-

${ }^{8}$ The $C_{\ell}$ and $C_{\ell}^{\text {cross }}$ are computed using HEALPix (Górski et al. 2005) using the standard definition

$$
C_{\ell}=\frac{1}{2 \ell+1} \sum_{m}\left|a_{\ell m}\right|^{2},
$$

and

$$
C_{\ell}^{\mathrm{cross}}=\frac{1}{2 \ell+1} \sum_{m} a_{\ell m} b_{\ell m}^{*},
$$

where $a_{\ell m}$ and $b_{\ell m}$ are the coefficients of the decomposition into spherical harmonics of two maps. We do this by evaluating the maps at the highest resolution of the Green et al. (2015) map $\left(N_{\text {side }}=2048\right)$. The pixelation affects the power spectra at the largest $\ell \gtrsim 2 \times 10^{3}$; we do not attempt to correct for this here. 

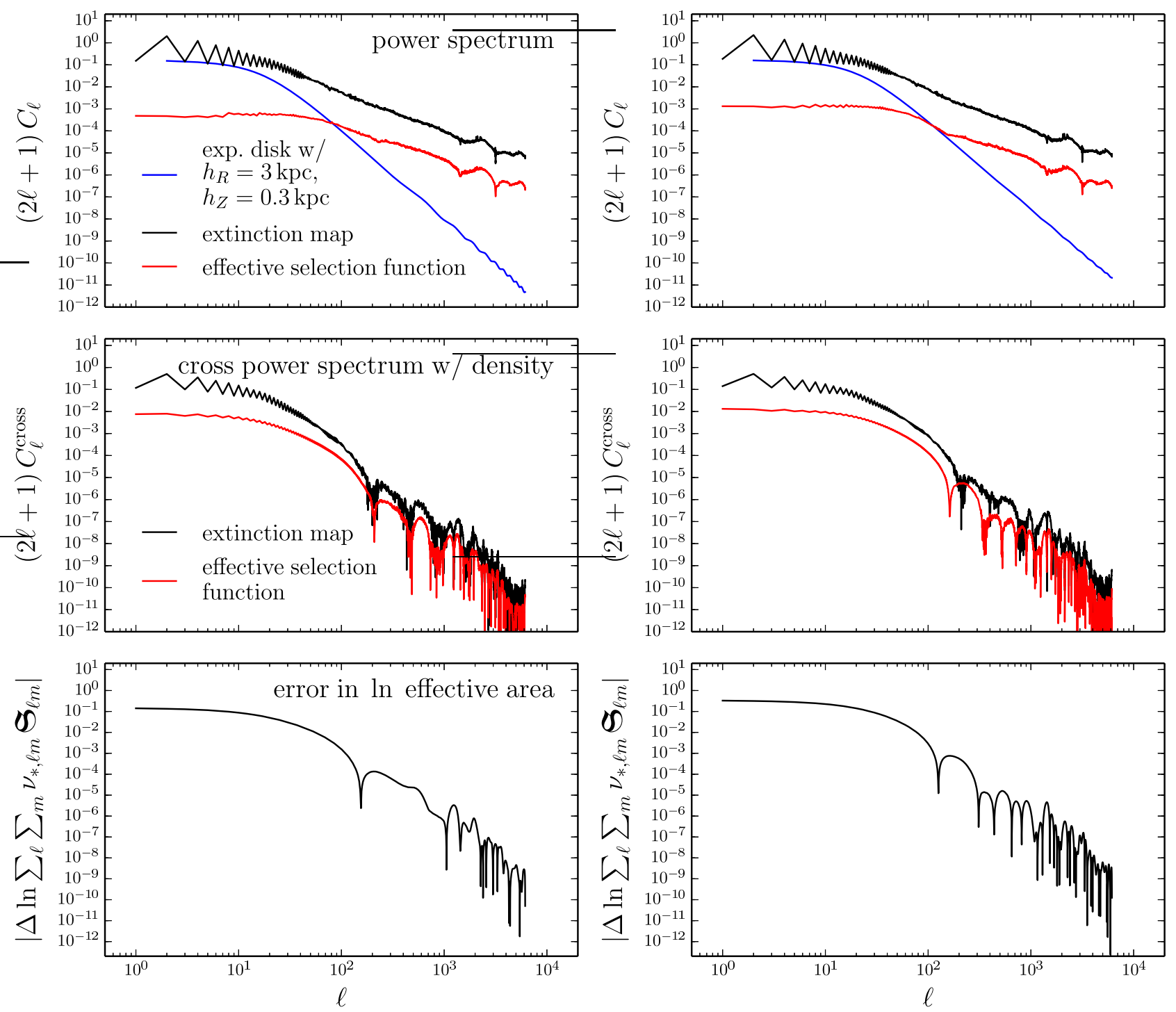

FIG. 3.- Top panels: Power spectrum of an exponential disk (blue; $h_{R}=3 \mathrm{kpc}, h_{Z}=0.3 \mathrm{kpc}$ ), the combined extinction map (see the Appendix) (black), and the selection function of a Gaia-like RC sample ( $\leq G \leq 20$; red) at $5 \mathrm{kpc}$ (left panels) and $6.3 \mathrm{kpc}$ (right panels). Middle panels: Cross power-spectra of the density with the extinction map (black) and the selection function (red). Bottom panels: error in calculating the natural logarithm of the effective area when only including terms up to spherical degree $\ell$ in the calculation: the effective area is the cross-correlation of the density and the selection function. The pixelization of the extinction maps affects the power spectra at $\ell \gtrsim 2 \times 10^{3}$. The density acts as a low-pass filter on the selection function, whose integrated power would otherwise only slowly converge when going to smaller scales.

ror in computing the effective survey volume when only including terms up to spherical degree $\ell$ in the calculation, that is, when limiting the resolution to $\approx 180^{\circ} / \ell$.

When performing a likelihood-based inference we need to be able to compute the relative log likelihood of models within $-2 \Delta \ln \mathcal{L}=\Delta \chi^{2} \approx \mathcal{O}(1)$ of each other to a precision that is much smaller than 1. This is such that errors in the likelihood computation do not affect the uncertainty assigned to model parameters (which are roughly assigned based on $\left.\Delta \chi^{2} \approx \mathcal{O}(1)\right)$. Because the effective volume normalizes the probability for each data point (see equation [4]), this requires us to compute the logarithm of the effective volume of models within $\Delta \chi^{2} \approx \mathcal{O}(1)$ to a precision much better than $1 / N$ for a sample of $N$ data points. The bottom panels of Figure 3 display the absolute precision in the effective vol- ume (that is, the precision of a single effective-volume calculation); if this is smaller than $1 / N$, then the relative precision - the precision of the logarithmic difference between the effective volumes of two similar models - will also be computed precisely enough. For a Gaia-sized survey of $\mathrm{RC}$ stars, $N \approx 10^{6}$, which requires an extinction map at a resolution of $\lesssim 10^{\prime}(\ell \approx 1,000)$. This is close to the resolution in $3 \mathrm{D}$ extinction maps that exists in current maps (see the discussion in the Appendix). Extrapolating the behavior at $\ell \lesssim 2 \times 10^{3}$ - where it is not affected by the pixelization of current extinction maps - in the bottom panels of Figure 3, we find that the full Gaia data set $\left(N \approx 10^{9}\right)$ requires a resolution $\lesssim 2^{\prime}$, which is at the limit of current extinction maps. Modest improvements in the currently available 3D extinction maps will therefore suffice for computing the effective area for any 


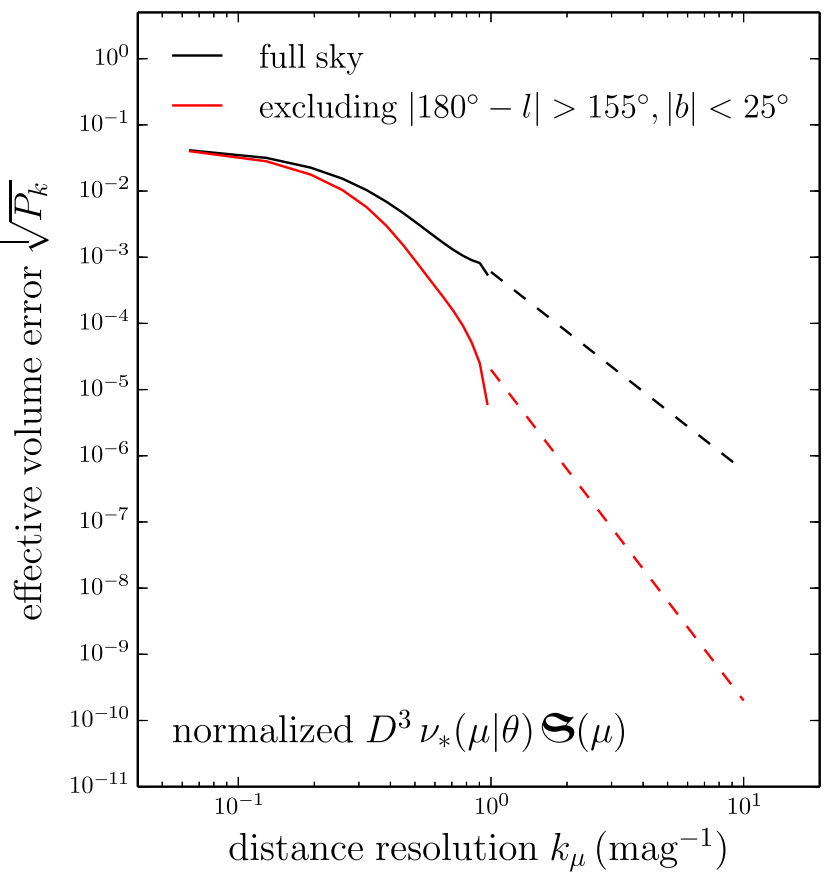

FIG. 4.- Power spectrum of the effective area as a function of distance modulus, computed for an exponential disk $\left(h_{R}=3 \mathrm{kpc}\right.$, $h_{Z}=0.3 \mathrm{kpc}$ ) and the selection function of a Gaia-like RC sample $(3 \leq G \leq 20)$. This power spectrum can be interpreted as the error made in the computation of the effective volume as a function of the limited distance sampling of 3D extinction maps. The black curve displays the full-sky effective area and the red curve excludes a rectangular inner $25^{\circ} \times 25^{\circ}$ box. The error in the effective volume due to the poor distance sampling of current extinction maps is $\approx 10^{-3}$ over the full sky, but dominated by the highly-extinguished inner Galaxy; excluding the central region, currently the error is $\approx$ $10^{-5}$. Extrapolating the trends with resolution (dashed lines), an order of magnitude improvement in the distance resolution should lead to errors $\lesssim 10^{-6}$ over the full sky and $\lesssim 10^{-10}$ excluding the central region, which is good enough for large-scale Gaia analyses.

smooth model for the phase-space distribution of stars constrained using Gaia data. Analyses using samples with small sizes do not gain from using a high-angularresolution effective selection function; an analysis such as that presented in the bottom panels of Figure 3 can be used to determine a good resolution to degrade the effective selection function to, to minimize the computational cost for computing the effective selection function.

Computing the effective volume requires us to integrate the effective area over distance (see equation [10]). While the angular resolution of $3 \mathrm{D}$ extinction maps is high, the distance resolution of these maps is poor due to, for example, uncertainties in the distances to the stellar tracers used to determine the extinction. The spatial sampling in distance modulus of the Green et al. (2015) and Marshall et al. (2006) maps is $\approx 0.5 \mathrm{mag}$, although we note that this is undersampled by a factor of $\approx 2$, in that a finer distance sampling is possible for the same data. This resolution is much worse than the angular resolution of these maps ( $\approx 10^{\prime}-15^{\prime}$; see the Appendix), which corresponds to a relative distance resolution of less than $1 \%$. However, because extinction along a line of sight is a cumulative quantity, the behavior of extinction with distance is typically smoother than the angular dependence. The impact of the distance resolution on the error in the effective volume should then be less severe.

To assess the error in the effective volume due to dis- tance resolution, we have computed the effective area for the same exponential disk model and Gaia-like RC selection as above at all of the distance moduli of the combined extinction map of the Appendix. Figure 4 shows the power spectrum - calculated using a standard periodogram estimate - of the effective area as a function of distance modulus (this includes an extra factor of distance because of the transformation between distance and distance modulus). The power spectrum at a given resolution is the approximate error due to the ignorance of sub-resolution fluctuations in the area; this approximation works well because the power spectrum steeply declines with increasing $k$. An estimate of the error in computing the effective volume using Simpson's rule agrees with the power spectrum estimate. The black curve in Figure 4 displays the power spectrum using the full-sky effective area. This leads to an error on the $0.5 \mathrm{mag}$ spatial sampling of $\approx 10^{-3}$. An investigation of the angular dependence of the integrand reveals that much of this error comes from the inner region of the MW, where both the extinction and the density of an exponential disk are high and small-scale structure in the extinction has a significant impact on the effective volume. Excluding the inner regions (the red curve in Figure 4) removes much of the error and leads to an error of $\approx 10^{-5}$. The error also decreases about twice as fast with increasing resolution outside of the inner regions. Increasing the distance sampling above that of Green et al. (2015) by a factor of ten, which can be achieved within a few years using the precise Gaia parallaxes for many stellar-extinction tracers, leads to errors of $\approx 10^{-6}$ (full sky) and $\approx 10^{-10}$ (excluding the central regions). The latter is good enough to allow sensitive measurements of the disk's structure and asymmetries using the full Gaia catalog, but studying the inner regions with large Gaia samples will be challenging.

The discussion so far has focused on angular and distance precision. However, accuracy is as important for disentangling intrinsic structural properties of the Milky Way's stellar distribution from those of the dust distribution. In Figure 5 below, we compare the distribution of near-infrared (NIR) extinction in a few lines of sight for four existing $3 \mathrm{D}$ extinction maps. While overall the agreement is good, differences between different maps are much larger than the precision of each of the maps. In particular, extinction maps constructed largely using optical data (Sale et al. 2014 and Green et al. 2015) disagree with IR-based maps in regions of high extinction. Because the high-extinction regime is the most important for the error in the effective volume, future extinction maps will need to incorporate IR data to properly sample this regime.

\section{THE EFFECTIVE SURVEY VOLUME FOR PENCIL-BEAM SURVEYS}

\subsection{Formalism}

In principle the same formalism as presented above could be applied to a pencil-beam survey. However, the sharp edges of each celestial pointing in pencil-beam surveys would introduce significant ringing in the effective selection function at each order, while apodizing the boundary of each field would require much data to be discarded. Pencil-beam surveys are closer to the case of 

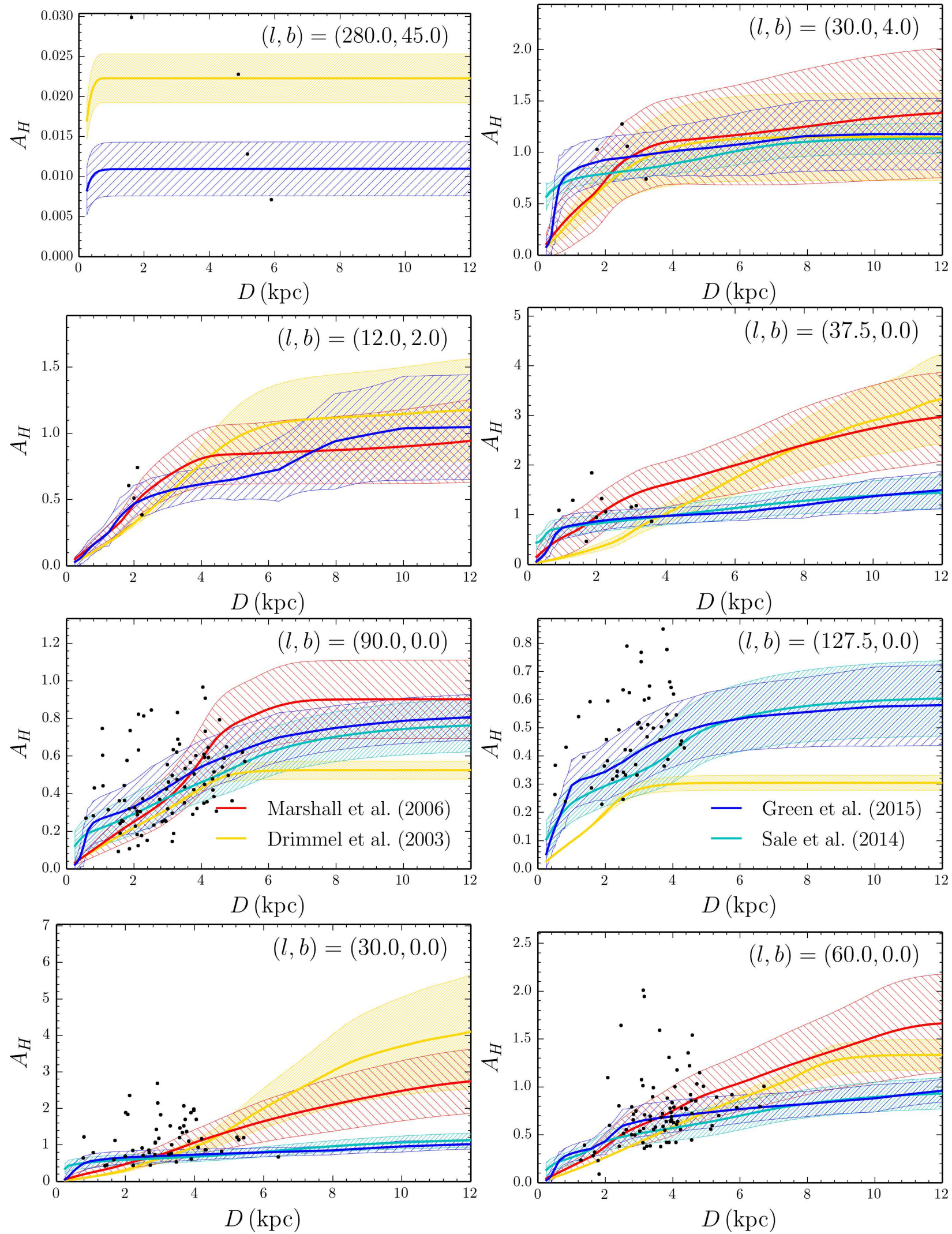

FIG. 5.- Distribution of extinction $A_{H}$ as a function of distance for four different three-dimensional extinction maps and eight representative APOGEE pointings. The solid line for each map displays the mean extinction over the area of an APOGEE pointing (which have radii of $1.49^{\circ}$, except for the $(l, b)=(12.0,2.0)$ pointing that has a radius of $\left.1^{\circ}\right)$ and hatched regions demonstrate the 1 sigma range of the distribution. The dots are data points from the APOGEE-RC catalog. Overall the different extinction maps agree well with each other and with the data (the large discrepancies between the Sale et al. (2014)/Green et al. (2015) and Drimmel et al. (2003)/Marshall et al. (2006) maps are beyond where the former maps are considered reliable). 
a set of delta-function pointings on the sky, where we would compute the effective volume by summing over these pointings.

As an example of a pencil-beam survey we will consider the selection function of RC stars in the APOGEE survey. For this survey the survey selection function is accurately known (Bovy et al. 2014) and it is a function of the field and apparent $H$ magnitude (not corrected for extinction). That is, $S \equiv S$ (field, $H$ ). The RC is close to a standard candle in the NIR, with variations as a function of color and metallicity of $\approx 0.1$ mag. Nevertheless, we will continue to present the formalism for the general case where there are variations of the absolute magnitude with color $c$ (typically $c \equiv\left(J-K_{s}\right)_{0}$ for the NIR RC) and $[\mathrm{Fe} / \mathrm{H}]$. With APOGEE in mind, we use $H$ instead of the generic magnitude $m$ in this section and we again replace the arguments $(l, b, D, c,[\mathrm{Fe} / \mathrm{H}])$ of $H$ by [.] in many expressions to avoid notational clutter.

The effective volume for a pencil-beam survey can be written as a sum over field locations

$$
\int \mathrm{d} O \lambda(O \mid \theta)=\sum_{\text {fields }} \int \mathrm{d} D D^{2} \nu_{*}([X, Y, Z](D, \text { field }) \mid \theta) \times \iint \mathrm{d} c \mathrm{~d}[\mathrm{Fe} / \mathrm{H}] \rho(c,[\mathrm{Fe} / \mathrm{H}]) \times \iint \mathrm{d} l \mathrm{~d} b \cos b S(\text { field }, H[\cdot]),
$$

where we have assumed that the density $\nu_{*}(l, b, D)$ is sufficiently smooth that it can be considered constant over the extent of the field. When this is not the case, $\nu_{*}(l, b, D)$ could be expanded in terms of basis functions around the center of each field in much the same way as in equation (6), which would introduce higher-order effective selection functions below, in the same manner as in equation (8). We further assume that the absolutemagnitude spread of the stellar tracer does not have significant variations within a field; field-to-field variations could be included, but are also neglected here.

From the expression in equations (13), it is clear that the three-dimensional distribution of extinction only enters in the integration over $(l, b)$. We then again introduce the effective selection function $\boldsymbol{S}$. For a standard candle, $\boldsymbol{S}$ is the selection function averaged over $(l, b)$

$$
\mathbf{s}(\text { field }, D)=\iint \frac{\mathrm{d} l \mathrm{~d} b}{\Omega} \cos b S(\text { field }, H[l, b, D]),
$$

(standard candle; 14)

where $\Omega$ is the area of the $(l, b)$ integration. If the tracer stellar population is not a standard candle, we absorb the integration over $(c,[\mathrm{Fe} / \mathrm{H}])$ into the definition of the effective selection function

$$
\begin{aligned}
& \boldsymbol{s}(\text { field }, D)=\iint \mathrm{d} c \mathrm{~d}[\mathrm{Fe} / \mathrm{H}] \rho(c,[\mathrm{Fe} / \mathrm{H}]) \\
& \times \iint \frac{\mathrm{d} l \mathrm{~d} b}{\Omega} \cos b S(\text { field }, H[l, b, D, c,[\mathrm{Fe} / \mathrm{H}]])
\end{aligned}
$$

(general case; 15)

This integration over $(c,[\mathrm{Fe} / \mathrm{H}])$ can again be easily performed using Monte Carlo integration.

With this definition of the effective selection function, the effective volume becomes simply

$$
\begin{aligned}
& \int \mathrm{d} O \lambda(O \mid \theta)= \\
& \sum_{\text {fields }} \Omega \int \mathrm{d} D D^{2} \nu_{*}([X, Y, Z](D, \text { field }) \mid \theta) \mathbf{S}(\text { field }, D) .
\end{aligned}
$$

\subsection{The effective selection function of the APOGEE-RC sample}

The APOGEE selection function is a constant $S$ (field, $k$ ) within each bin $k$ of a small number of magnitude bins $\left[H_{\min , k}, H_{\max , k}\right]$ (Zasowski et al. 2013; Bovy et al. 2014). Therefore, we can simplify $\boldsymbol{S}$ to

$$
\mathbf{S}(\text { field }, D)=\sum_{k} S(\text { field }, k) \frac{\Omega\left(H_{\min , k}-H_{0}(D)<A_{H}(l, b, D)<H_{\max , k}-H_{0}(D)\right)}{\Omega_{f}},
$$

for a standard candle with $H_{0}=M_{H}+\mu$, where $M_{H}$ is the absolute magnitude of the standard candle and $\mu$ is the distance modulus; $\Omega\left(H_{\min , k}-H_{0}<\right.$ $\left.A_{H}(D)<H_{\max , k}-H_{0}\right)$ is the area of the field with $A_{H}$ between the given boundaries and $\Omega_{f}$ is the total area of the field (not all APOGEE fields have the same area). For a non-standard candle we additionally integrate over $(c,[\mathrm{Fe} / \mathrm{H}])$, which in the APOGEE-RC case are $\left(\left[J-K_{s}\right]_{0},[\mathrm{Fe} / \mathrm{H}]\right)$. In this expression, we have suppressed the dependence of $A_{H}$ on $(l, b)$ for clarity. The APOGEE selection function $S$ and effective selection function $\boldsymbol{S}$ for a standard candle or any tracer with a known $M_{H}$ distribution is implemented as part of the apogee Python package at http://github.com/jobovy/apogee as part of its apogee. select.apogeeSelect module.

Thus, at any given distance the effective selection function only depends on a smoothed version of the twodimensional extinction distribution at that distance: the fractional area of the field in wide $(\approx 1 \mathrm{mag})$ bins in $A_{H}(D)$. For the RC sample that we use here the dependence of $H_{0}$ on $\left(\left[J-K_{s}\right]_{0},[\mathrm{Fe} / \mathrm{H}]\right)$ is weak $(\lesssim 0.1 \mathrm{mag}$; 
see Figure 3 of Bovy et al. 2014), such that we can treat the RC as a standard candle. The weak dependence of the $\mathrm{RC}$ absolute magnitude implies that any density or phase-space fits are only very weakly dependent on the color-metallicity distribution of stars in the sample.

To illustrate the pattern of extinction that affects APOGEE observations, we can interpret the extinction displayed in Figure 1 as the $H$-band extinction between 0 and $1.1\left(A_{G} / A_{H} \approx 5.1\right)$. This upper limit corresponds to the maximum extinction for which we can see the $\mathrm{RC}$ to $\approx 4.5 \mathrm{kpc}$ in a medium-deep APOGEE pointing $(H<12.8)$. This gives a sense of the volume over which the RC can be mapped with APOGEE. RC stars can be seen to $4 \mathrm{kpc}$ over almost the whole sky, while at $5 \mathrm{kpc}$ and further much of the disk region is obscured. APOGEE has deep pointings (down to $H<13.8$ ) along a few lines-of-sight that allow for some of this highlyextinguished structure to be penetrated.

In Figure 5 we demonstrate the range of extinction values in eight APOGEE pointings for four different threedimensional extinction maps. The default extinction model that we use is that of Green et al. (2015), which is displayed in blue. Each curve shows the mean extinction within the APOGEE pointing and the hatched regions display the $1 \sigma$ range of the extinction distribution. Other three-dimensional extinction maps are compared to our default model: the yellow curve displays the extinction model of Drimmel et al. (2003), which is a fully-sky map with an angular resolution of $\approx 20^{\prime}$; the red curve shows the 3D map of Marshall et al. (2006) which is available over $-100^{\circ} \leq l \leq 100^{\circ}$ and $|b| \leq 10^{\circ}$; and the cyan curve gives the map of Sale et al. 2014, available over $30^{\circ} \leq l \leq 215^{\circ}$ and $|b| \leq 5^{\circ}$. The agreement between the recent extinction maps of Sale et al. (2014) and Green et al. (2015) is remarkably good, but they both typically give smaller extinction close to the Galactic plane than the Marshall et al. (2006) map. The APOGEE-RC data themselves provide good estimates of extinction values at the range of distances included in our sample and our data are displayed as black dots. Overall the agreement between the extinction maps and our data is good; however, our extinction vs. distance data do not allow us to unambiguously prefer the Marshall et al. (2006) map over the Green et al. (2015) map at low Galactic latitudes.

While the differences between the different 3D extinction maps can be quite substantial, especially at large distances, they only matter for any density inference in as much as they lead to different effective selection functions $\mathbf{S}$ (field, $D$ ). Figure 6 demonstrates the effective selection function for the same eight pointings as displayed in Figure 5. At Galactic latitudes above a few degrees, the different maps lead to similar effective selection functions, even though the extinction can be substantial ( $A_{H} \approx 1$ near the faint end of the survey). We have included one APOGEE pointing in the inner Galaxy at $(l, b)=(12,2)$ : these locations were only observed down to $H<11$ and therefore the effective selection function only reaches to $\approx 3 \mathrm{kpc}$; the different extinction maps all return the same $\boldsymbol{\delta}$ for such inner-Galaxy pointings. Within a few degrees of the mid-plane, larger differences occur between the effective selection function calculated using the Marshall et al. (2006) map and the Green et al. (2015)/Sale et al. (2014) maps, especially at $l \lesssim 60^{\circ}$.
All but one of the curves in Figure 6 are computed assuming that the $\mathrm{RC}$ is a standard candle. The dashed green line demonstrates what happens when we relax this assumption for the Green et al. (2015) map. For all but the least obscured pointings, the small $\approx 0.1$ mag width of the $\mathrm{RC}$ absolute magnitude has no effect on the effective selection function, essentially because the small-scale structure in the extinction map washes out this small spread. Only at high Galactic latitude is the extinction small enough that the small $M_{H}$ spread in the $\mathrm{RC}$ can affect the effective selection function.

We estimate the error in the calculation of the effective volume due to the distance integration in the same manner as for the Gaia example above for the eight fields of Figures 5 and 6 . We find that the current error is $\approx$ few $\times 10^{-3}$, decreasing by $\approx 10^{-2}$ per decade of improved distance resolution. These errors are larger than for the Gaia example above, mainly because of the much narrower magnitude bins employed by APOGEE and due to APOGEE's focus on the mid-plane, where the extinction has more small-scale structure. Analyses using hundreds of tracers are therefore unaffected by the current distance distance sampling. Using larger samples may lead to systematic uncertainties due to the limited distance sampling in current extinction maps, but again the relevant error is the relative error between models that fit the data similarly well $\left(\Delta \chi^{2}=\mathcal{O}(1)\right)$ and this is likely much smaller than the absolute error discussed here. Mock-data tests for an APOGEE-like sample of 20,000 stars by Bovy et al. (2016) indicate that such samples are also currently unaffected by the distance resolution. As discussed above, improvements in the distance resolution by about a factor of 10 are likely in the near future, such that spectroscopic samples close to the midplane of $\approx 10^{6}$ stars should be able to be analyzed; for spectroscopic surveys that avoid the mid-plane (i.e., optical surveys) the requirements are less severe.

\section{CONCLUSIONS}

Motivated by the advent of near-infrared spectroscopic surveys and Gaia, we have discussed likelihood-based phase-space inference in the MW in detail in this paper, focusing in particular on the effects of interstellar extinction. Central to this inference is the effective survey volume, which acts as a normalization factor in the likelihood. In principle the computation of this effective volume is straightforward: one simply integrates the density at $(l, b, D)$ multiplied by the selection function, which includes the effects of extinction, over the full MW volume. In practice, this integration is expensive and the uncertainties due the angular and distance resolution in the extinction map have been difficult to assess.

In $\S 3$, we have introduced a novel formalism that introduces the effective selection function to assess the impact of angular and distance resolution on the computation of the effective volume for a large-area survey such as Gaia. We have found that the angular resolution of current extinction maps is sufficient to analyze Gaia samples consisting of millions of stars in terms of smooth, axisymmetric density distribution. Only modest and realistic improvements in the angular resolution are necessary to analyze the full Gaia data set $\left(\approx 10^{9}\right.$ stars; in case one is so inclined). More limiting than the angular resolution is the distance sampling of current 

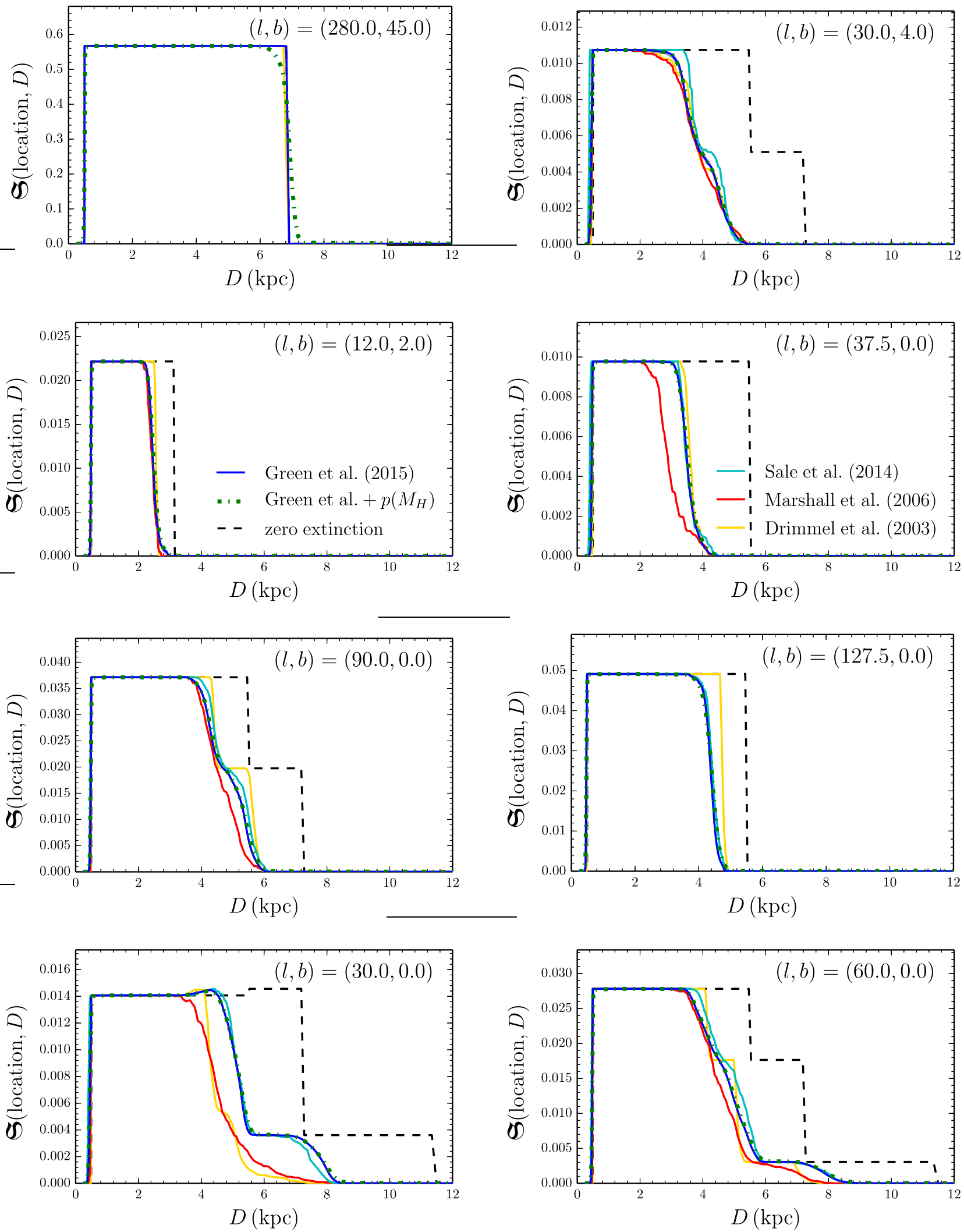

FIG. 6.- Effective selection function $\mathbf{S}$ (location, $D$ ) for eight APOGEE pointings. The dashed line displays the raw selection function, which is a piecewise-constant function over one, two, or three magnitude ranges, translated from $H$ to distance assuming $M_{H}=-1.49$ for the RC. The blue solid line shows the effective selection function calculated using the Green et al. (2015) 3D extinction map assuming that the RC is a standard candle, while the dot-dashed green line takes into account the spread in $M_{H}$ in the RC for the whole APOGEERC sample. The uncertainty in this effective selection function as calculated from the Green et al. (2015) MCMC samples is smaller than the width of the line. The effective selection function for three other extinction maps is also shown where these maps are available (Drimmel et al. 2003: full sky; Marshall et al. 2006; $-100^{\circ} \leq l \leq 100^{\circ}$ and $|b| \leq 10^{\circ}$; Sale et al. 2014: $30^{\circ} \leq l \leq 215^{\circ}$ and $|b| \leq 5^{\circ}$ ). The Marshall et al. (2006) map generally leads to a shallower effective selection function, because it has on average higher extinction (see Figure 5). The agreement between the two most recent extinction maps (Sale et al. 2014 and Green et al. 2015) is remarkably good. 
maps. The current distance sampling is about $0.5 \mathrm{mag}$, or about $25 \%$ in distance, which causes an uncertainty in the effective volume of $\approx 10^{-3}$ to $10^{-5}$, depending on how much of the inner MW is included. From an analysis of the small-scale structure in the distance integration, we expect this uncertainty to decrease by three orders of magnitude or five (excluding the inner MW) for an improvement in the distance resolution by a factor of ten. Such an improvement appears plausible with the precise Gaia distances for large numbers of stellar tracers of the extinction and allows sensitive analyses of the disk's structure with the full Gaia data set. Analyzing the nonaxisymmetric component of the stellar disk that is less smooth than the exponential disk models considered here would place somewhat more stringent conditions on the angular and distance resolution of extintion maps; these can be evaluated with a similar formalism as introduced here. Extinction maps also need to attain a higher accuracy, because current extinction maps differ by much more than their stated uncertainties in regions of high extinction. This requires a larger contribution from IR data in the creation of $3 \mathrm{D}$ extinction maps.

We have also discussed the formalism for phasespace inference of non-contiguous, spectroscopic surveys. An application of this formalism is given in Bovy et al. (2016), who analyze the density distribution of abundance-selected populations using RC stars in APOGEE. We have demonstrated that the 3D extinction map can be fully and efficiently taken into account in likelihood-based phase-space inferences using an effective selection function under the assumption that the density is close to constant within each spectroscopic pointing.

Our formalism makes it clear that in both the contiguous and the non-contiguous case the small-scale structure in the 3D extinction map is filtered by the smooth density field. This is because the effective survey volume is essentially the cross-correlation of the smooth (phase-space) density and the effective selection function; as the density is very smooth, this cross-correlation is effectively a low-pass filtering of the effective selection function, which removes the influence of the patchy extinction map.

Progress in obtaining better 3D extinction maps in the future will require a balance between angular and distance resolution because of the finite number of stars available to use as tracers. Essentially, one can use larger angular bins to obtain a larger number of stars to infer the distance dependence of the extinction, although in practice a better approach is to require a high degree of correlation between adjacent, small angular bins or by using Gaussian processes (e.g., Sale \& Magorrian 2014). From our analysis we can conclude that improving the distance resolution-especially in the highextinction, inner Galaxy - is much more important for studies of Galactic structure than improving the angular resolution. This is especially important for future analyses that aim to take advantage of the full Gaia catalog to tease out small signals of, e.g., phase-space asymmetries. Such analyses require the best smooth background models and the ability to compute the background model to an accuracy of $\approx 10^{-9}$.

It is a pleasure to thank the anonymous referee and Wilma Trick for helpful comments. Some of the results in this paper have been derived using the HEALPix (Górski et al. 2005) and healpy packages. J.B. received support from a John N. Bahcall Fellowship, the W.M. Keck Foundation, and the Natural Sciences and Engineering Research Council of Canada. H.W.R. received funding for this research from the European Research Council under the European Union's Seventh Framework Programme (FP 7) ERC Grant Agreement n. [321035]. J.B. and H.W.R. acknowledge the generous support and hospitality of the Kavli Institute for Theoretical Physics in Santa Barbara during the 'Galactic Archaeology and Precision Stellar Astrophysics' program, where some of this research was performed.

\section{APPENDIX}

\section{A THREE-DIMENSIONAL EXTINCTION MAP OVER THE FULL SKY}

In recent years a number of three-dimensional maps of the integrated extinction out to many kpc based on modeling stellar photometry have appeared (e.g., Marshall et al. 2006; Sale et al. 2014; Green et al. 2015). None of these cover the full sky. To illustrate the formalism for accounting for the effect of extinction on the volume selection of stellar surveys presented in this paper and to make projections for Gaia, we require a full-sky, three-dimensional extinction map.

We perform a simple combination of the extinction maps of Marshall et al. (2006) (based on 2MASS data) and Green et al. (2015) (based on Pan-STARRS and 2MASS data) and fill in the remaining parts of the sky with the map of Drimmel et al. (2003), a three component analytic model for the dust distribution fit to the COBE DIRBE data and normalized to the map of Schlegel et al. (1998). Bovy et al. (2016) found by modeling the observed counts of RC stars near the midplane in APOGEE that the map of Marshall et al. (2006) performs better than that of Green et al. (2015) where they overlap, essentially because the latter underestimates the amount of extinction in regions of high extinction due to the paucity of main-sequence stars that they primarily use, while Marshall et al. (2006) mainly employ giant stars that are visible to larger distances. We do not use the map of Sale et al. (2014), as it led to inferior fits of the APOGEE stellar densities.

Specifically, we follow the hierarchical HEALPix format of Green et al. (2015) and evaluate the Marshall et al. (2006) map, which exists over the range $-100^{\circ} \leq l \leq 100^{\circ}$ and $|b| \leq 10^{\circ}$, on a grid of HEALPix pixel centers with $N_{\text {side }}=512$ or an approximate resolution of $7^{\prime}$. We employ this resolution to slightly oversample the native resolution of $15^{\prime}$. We then add the hierarchical map of Green et al. (2015), which has a variable angular resolution of $\approx 4^{\prime}$ to $14^{\prime}$, outside of the Marshall et al. (2006) area. The remaining area of the sky, mainly near the south celestial pole, is filled in with the map of Drimmel et al. (2003) at a HEALPix resolution of $N_{\text {side }}=256$, which again somewhat oversamples the resolution of the Drimmel et al. $(2003)$ map $\left(\approx 20^{\prime}\right)$. We also fill in parts of $N_{\text {side }}=256$ pixels that are partially 
covered by the other two maps.

The resulting map of $A_{G}$ at $5 \mathrm{kpc}$ is displayed in Figure 1 . We use $A_{G} / E(B-V)=2.35$ (Bovy et al. 2014). While this map is not perfect and the effect of stitching together the three different extinction maps is clearly visible, this map does an adequate job of describing the extinction over the full sky and is good enough for our purposes here. The most problematic region is the region of the Galactic plane covered by neither the Marshall et al. (2006) or the Green et al. $(2015) \operatorname{map}\left(250^{\circ} \lesssim l \lesssim 260^{\circ}\right)$, but in the high-latitude regions filling in uncovered regions with the model of Drimmel et al. (2003) performs well.

To aid in the comparison of different 3D extinction maps we have made a code publicly available at http://github.com/jobovy/mwdust

This code downloads and installs all of the necessary data and code to evaluate the extinction maps of Schlegel et al. (1998), Drimmel et al. (2003), Marshall et al. (2006), Sale et al. (2014), and Green et al. (2015) using a common interface.

\section{REFERENCES}

Bovy, J., Rix, H.-W., Liu, C., et al. 2012, ApJ, 753, 148

Bovy, J., Nidever, D. L., Rix, H.-W., et al. 2014, ApJ, 790, 127

Bovy, J., Rix, H.-W., Schlafly, E. F., et al. 2016, ApJ, 823, 30

Bressan, A., Marigo, P., Girardi, L., et al. 2012, MNRAS, 427, 127

Drimmel, R., Cabrera-Lavers, A., \& López-Corredoira, M. 2003, A\&A, 409, 205

Górski, K. M., Hivon, E., Banday, A. J., et al. 2005, ApJ, 622, 759

Green, G. M., Schlafly, E. F., Finkbeiner, D. P., et al. 2015, ApJ, 810,25

Hayden, M. R., Bovy, J., Holtzman, J. A., et al. 2015, ApJ, 808, 132
Jordi, C., Gebran, M., Carrasco, J. M., et al. 2010, A\&A, 523, A48

Marshall, D. J., Robin, A. C., Reylé, C., Schultheis, M., \& Picaud, S. 2006, A\&A, 453, 635

Rix, H.-W. \& Bovy, J. 2013, A\&A Rev., 21, 61

Sale, S. E. \& Magorrian, J. 2014, MNRAS, 445, 256

Sale, S. E., Drew, J. E., Barentsen, G., et al. 2014, MNRAS, 443, 2907

Schlegel, D. J., Finkbeiner, D. P., \& Davis, M. 1998, ApJ, 500 525

Zasowski, G., Johnson, J. A., Frinchaboy, P. M., et al. 2013, AJ, 146,81 